OPEN ACCESS

Edited and reviewed by: Jordi Figuerola Estación Biológica de Doñana, Spain

${ }^{*}$ Correspondence: Ann V. Hedrick avhedrick@ucdavis.edu

Specialty section:

This article was submitted to Behavioral and Evolutionary Ecology, a section of the journal

Frontiers in Ecology and Evolution

Received: 08 December 2016

Accepted: 24 February 2017

Published: 14 March 2017

Citation:

Hedrick AV (2017) Editorial: The

Development of Animal Personality.

Front. Ecol. Evol. 5:14.

doi: 10.3389/fevo.2017.00014

\section{Editorial: The Development of Animal Personality}

\author{
Ann V. Hedrick* \\ Neurobiology, Physiology and Behavior and Animal Behavior Graduate Group, University of California Davis, Davis, CA, USA
}

Keywords: development, animal personality, boldness, exploratory behavior

\section{Editorial on the Research Topic}

\section{The Development of Animal Personality}

Over the past 20-odd years, researchers in behavioral ecology have increasingly focused on consistent individual differences in behavior, also known as animal personality, and their causes and consequences. In 2010, Stamps and Groothuis published a highly influential paper drawing attention to the importance of development in the study of animal personality and lamenting the lack of research on this topic (Stamps and Groothuis, 2010). Since then, researchers have increasingly focused on this gap in our knowledge. The current volume draws together nine papers with a variety of approaches on the development of personality, and provides a glimpse into the current state of the art.

Four of the papers are non-empirical (data-free). Davis et al. directly address the issue of datafree papers on animal personality. They find that data-free papers comprise $20 \%$ of the papers on animal personality in the last decade, and argue that these papers are vitally important because they can synthesize current knowledge, integrate diverse fields in novel ways, and/or identify important future directions for the field.

Stamps and Krishnan discuss a Bayesian model they have constructed, using it to explain why we would expect individual differences in the developmental trajectories of personality traits, even if all animals are raised under the same conditions. They also examine commonly used experimental protocols (within-individual and replicate individual) and explain why replicate individual designs are better suited to provide estimates of the developmental plasticity of behavioral traits. Finally, they advocate using a Bayesian approach that assumes differences between individuals in the information provided by immediate and distant ancestors to offer insights into the effects of genes, epigenetic factors, maternal effects and personal experiences on the development of personality.

To date, research on animal personality has seldom considered social partners as important determinants of personality. Niemela and Santostefano address how social partners might affect behavioral variation and impose selection on animal personality, a process they refer to as "social carry-over effects." They point out that such effects can create variation among individuals in nonsocial behaviors, such as boldness, if the proximate mechanisms for these effects are irreversible. They also suggest approaches for studying the attributes and evolutionary outcomes of social carry-over effects on non-social behaviors.

Personality at the level of the group has also been a neglected topic. Bengston and Jandt focus on group-level, or "collective" personality, which they define as an emergent behavioral phenotype displayed at the group level. They emphasize that just as individual behavior changes as the individual matures, so too can group-level behavior change as the group matures. They examine a variety of factors that can generate collective personalities, including the genetic composition of the group and the internal social environment of the group, and discuss ways to evaluate the development of collective personality. 
The remainder of the papers in this volume are empirical, and cover a range of taxa including arachnids, insects, fish and birds. Liedtke et al. examine the effects of environmental complexity on the development of personality in jumping spiders. Using a split-brood design with a non-enriched, socially enriched and physically enriched environment, they find that exploratory behavior is lowest in non-enriched spiders and highest in physically enriched spiders. Moreover, significant family by environment interactions suggest that families differ in their developmental plasticity.

Snell-Rood and Steck use cabbage white butterflies to test the hypothesis that slow versus fast exploration of the environment can affect the development of cognitive abilities, resulting in movement-cognition syndromes in adults. They find that genotypes with smaller thoraxes are slower fliers, better at learning and develop larger brains, and conclude that genetic variation in behaviors such as exploration or movement can affect the development of other behavioral traits resulting in suites of correlated behaviors.

DiRienzo et al. employ a different perspective, and ask whether exposure to pathogens during development creates or diminishes individual differences in behavior in a field cricket. Specifically, they examine whether exposure to a bacterial pathogen as a juvenile affects mean adult boldness, variation in individual boldness, and/or immune response. Surprisingly, they find that a single early exposure to a bacterial pathogen is sufficient to alter the expression of boldness in adults, in that it extinguishes repeatability in boldness for those crickets injected with the pathogen. However, the pathogen exposure has no influence on immune function itself or the boldness-immune function relationship.

In a related paper, Kortet et al. examine the effects of a trematode parasite on personality in Eurasian minnows in the field. The authors assess boldness and activity, and their repeatabilities, in fish from two rivers, one with predatory fish and parasites and the other with neither. They predict that fish in the high-predation, high-parasite river should be bolder (in order to grow more quickly) and more consistent. Their results show that these fish are indeed bolder, but are less active (perhaps

\section{REFERENCES}

Stamps, J., and Groothuis, T. (2010). The development of animal personality: relevance, concepts and perspectives. Biol. Rev. 85, 301-325. doi: 10.1111/j.1469-185X.2009.00103.x

Conflict of Interest Statement: The author declares that the research was conducted in the absence of any commercial or financial relationships that could be construed as a potential conflict of interest. because of the parasite), whereas fish in both populations are consistent in boldness and activity. As the authors observe, this is one of the first papers linking predation and parasitism risk to the development of animal personalities.

The final paper in the volume deals with another vertebrate, the Superb Fairy Wren. Hall et al. ask whether variation in life-history strategies is associated with consistent differences in behavior. They assess the relationship between survival and a number of risk-related behaviors. Their results indicate that "proactive" birds are more exploratory in novel artificial environments, move into rooms faster, are more active, are more likely to approach a mirror image, are less docile during handling, and have lower survival rates, conforming to the "pace of life" syndrome. Interestingly, wrens become more exploratory and active when in poorer condition or when environmental conditions are harsher.

In conclusion, this volume provides a road map for estimating the developmental plasticity of behavioral traits, gives approaches for studying social carry-over effects and ways to evaluate the development of collective personality, and offers examples of ways to measure the effects of physical and social enrichment on personality, the emergence of correlations between movement or exploration and cognition, the effects of immune challenges and predation/parasitism on the development of personality, and the relationship between risk-related personality traits and lifelong survival. Above all, the papers in this volume illustrate the diversity of approaches that have shed light on the development of animal personality since Stamps and Groothuis' landmark review in 2010, and raise important questions for future study.

\section{AUTHOR CONTRIBUTIONS}

The author confirms being the sole contributor of this work and approved it for publication.

\section{FUNDING}

AH was supported by NSF IOS-1558069 during the preparation of this editorial.
Copyright (c) 2017 Hedrick. This is an open-access article distributed under the terms of the Creative Commons Attribution License (CC BY). The use, distribution or reproduction in other forums is permitted, provided the original author(s) or licensor are credited and that the original publication in this journal is cited, in accordance with accepted academic practice. No use, distribution or reproduction is permitted which does not comply with these terms. 